\title{
Genetic Characterization of GRA6 Genes from Toxoplasma gondii from Pigs in Okinawa, Japan
}

\author{
Satoshi ZAKIMI ${ }^{1)}$, Hisako KYAN ${ }^{2)}$, Mamoru OSHIRO ${ }^{1)}$, Chihiro SUGIMOTO ${ }^{3)}$, Xuan XUENAN ${ }^{3)}$ and Kozo FUJISAKI ${ }^{3)}$ \\ 1) Okinawa Prefectural Institute of Animal Health, Kohagura 112, Naha, Okinawa 900-0024, 2) Okinawa Prefectural Meat Inspection \\ Office, Ozato 2015, Ozato, Okinawa 901-1202 and ${ }^{3)}$ National Research Center for Protozoan Disease, Obihiro University of Agriculture \\ and Veterinary Medicine, Inada-cho, Obihiro, Hokkaido 080-8555, Japan
}

(Received 13 October 2005/Accepted 8 June 2006)

\begin{abstract}
Toxoplasma gondii from pigs in Okinawa Prefecture was characterized by nested PCR-restriction fragment length polymorphism (RFLP) and DNA sequence analysis of the dense granule antigen GRA6 gene. By nested PCR, parasite DNA was detected in 33 out of 91 lymph node samples with lesions similar to those found in toxoplasmosis samples that had been collected from pigs at an abattoir. RFLP analysis with MseI was successfully conducted in 29 of 33 PCR-positive samples to group the isolates into one of the three genotypes of $T$. gondii. Genotyping of the 29 studied samples rendered the following results: 13 of type I (44.8\%), 14 of type II (48.3\%), and 2 of type III $(6.9 \%)$. The GRA6 genes of 12 Okinawa isolates were cloned and sequenced. Nine new nucleotide sequences were found, and nucleotide substitutions specific for the Okinawa isolates were found at 13 positions. Phylogenetic analysis indicated that all GRA6 sequences were divided into one of the 3 main groups, and Okinawa isolates of GRA6 genotypes II and III seemed to be closely related to the Beverley strain and the NED strain, respectively. The results from this study may provide basic and useful information for the analysis of the molecular epidemiology of $T$. gondii infection within Japan.
\end{abstract}

KEY WORDS: genotype, GRA6, Toxoplasma gondii.

J. Vet. Med. Sci. 68(10): 1105-1107, 2006

Toxoplasma gondii is a protozoan parasite that invades and resides in a wide range of host cells in all warm-blooded animals, including humans. Toxoplasmosis in humans is a potentially fatal disease of the developing fetus and immunocompromised (e.g., AIDS and transplant) patients and can cause severe ocular disease in otherwise healthy individuals $[4,7]$.

Many different genetic markers are now available for $T$. gondii strain typing. However, the allelic diversity detected for most of these markers is low ( 2 to 4 alleles). The vast majority of $T$. gondii strains and isolates studied until now fell into one of three distinct clonal lineages (referred to as types I, II, and III) [1,9]. PCR-restriction fragment length polymorphism (RFLP) analysis on single-copy genes is the most commonly used method for typing $T$. gondii isolates [3]. However, in Japan, there have been few reports regarding the prevalence of the genotypes of the parasite isolated from humans and animals, including pigs. It has been the general view that, in Europe and the U.S.A., pork is the major source of $T$. gondii infection in humans $[5,16]$.

Dense granule antigens (GRA) are parasitic molecules secreted to the parasitophorous vacuole [15]. These molecules are likely to be involved in intracellular survival and the exchange mechanism with the host cell. GRA6, a singlecopy gene [11], is more polymorphic than the other markers [6]. We have used the coding region of the GRA6 gene for polymorphism analysis and determined the lineage types of $T$. gondii associated with $T$. gondii infection in pigs in Okinawa, Japan.

For this purpose, genetic analysis of the GRA6 locus for the amplification of the DNA obtained directly from tissue samples containing the parasite was performed, omitting the preceding process of isolation in mice or cell culture.

DNA samples containing $T$. gondii DNA examined in this study were obtained directly from pig lymph nodes. From 2003 through 2005, a total of 91 lymph node (mainly mesenteric) samples with hemorrhagic or necrotic lesions suspected of toxoplasmosis were obtained from 91 pigs at an abattoir on Okinawa Island. Parasite DNA was extracted from the lymph node samples using a SepaGene kit (Viogene, U.S.A.) according to the manufacturer's protocol. The presence of $T$. gondii had been determined by microscopic examination of an acridine orange-stained stamp smear sample and two nested PCRs separately amplifying the 5' and 3' ends of the SAG2 gene, which encode the tachyzoite surface antigen p22, as described previously [10]. Fifty-four and 57 of 91 lymph node samples were microscopically positive and SAG2-PCR-positive, respectively.

Nested PCR was done to amplify the coding region of the GRA6 gene. PCR amplification was performed with $1 \mu \mathrm{l}$ of DNA template in $50 \mu \mathrm{l}$ of a reaction mixture containing 10 $\mathrm{mM}$ Tris- $\mathrm{HCl}$, pH 8.3; $50 \mathrm{mM} \mathrm{KCl} ; 2 \mathrm{mM} \mathrm{MgCl}_{2} ; 200 \mu \mathrm{M}$ dNTPs; $0.5 \mu \mathrm{M}$ each of oligonucleotide primers; 1.25 units of Taq DNA polymerase (Takara, Japan); and 50 pmol of each primer. All PCRs were performed in an automatic DNA thermal cycler (Model TP600 Takara). The first step of amplification was $5 \mathrm{~min}$ of denaturation at $94^{\circ} \mathrm{C}$. This step was followed by 35 cycles, with 1 cycle consisting of $30 \mathrm{~s}$ at $94^{\circ} \mathrm{C}, 60 \mathrm{~s}$ at the annealing temperature for each pair of primers, and $90 \mathrm{~s}$ at $72^{\circ} \mathrm{C}$. The final cycle was followed by an extension step of $7 \mathrm{~min}$ at $72^{\circ} \mathrm{C}$. The PCR primer pair was designed from the GRA 6 gene sequence [11], GRA6FO: 5'-GGCAAACAAAACGAAGTG-3' and 
GRA6RO: 5'-CGACTACAAGACATAGAGTG-3' (positions $223-240$ and 1166-1147, respectively), used in the initial PCR reaction at an annealing temperature of $54^{\circ} \mathrm{C}$. The resulting amplification products were diluted to $1 / 10$ in water, and a second amplification was performed with the internal primers described by Fazaeli et al. [6], GRA6R: 5'GTAGCGTGCTTGTTGGCGAC-3' and GRA6; 5'TACAAGACATAGAGTGCCCC-3' (positions 372-391 and $1162-1143$, respectively), using $1 \mu l$ of the diluted product as the template at an annealing temperature of $60^{\circ} \mathrm{C}$. All of the final PCR products were purified with a GeneClean kit (Bio 101, U.S.A.) and digested with MseI. The PCR products and the restriction fragments were analyzed by $1.5 \%$ agarose gel electrophoresis.

The purified PCR products of the GRA6 gene of the 13 representative samples were cloned into a pGEM T Easy vector (Promega, U.S.A.), and the nucleotide sequences were determined by using the ABI PRISM 3100 or 3700 Genetic Analyzer (Applied Biosystems, U.S.A.) with the big dye chain terminator kit (Applied Biosystem). The nucleotide sequences were applied to a Basic Local Alignment Search Tool (BLAST) on the DNA Data Bank of Japan (DDBJ) database for a homology analysis against other GRA6 genes. Phylogenetic analyses of the GRA6 gene sequences were performed by the neighbor-joining method [14] using CLUSTAL W [17], and the bootstrap probabilities of each node were calculated using 1,000 replications.

Amplification of the GRA6 gene by nested PCR was successful in 33 of 91 (36.3\%) DNA samples extracted from pig lymph nodes that possessed toxoplasmosis-like lesions.

In order to determine the GRA6 type of $T$. gondii included in the samples, the GRA6 PCR products were digested with $M s e I$, and their type was determined according to the restriction pattern [6]. Figure 1 shows the results of a typical experiment. Twenty-nine of 33 GRA6 positive cases were successfully classified into one of the three types, rendering the following results: 13 of type I (44.8\%), 14 of type II (48.3\%), and 2 of type III (6.9\%). Recently, we also determined the prevalence of the 3 strain types of $T$. gondii from pigs in Okinawa by nested PCR-RFLP analysis of the $S A G 2$ locus. The frequencies of the $S A G 2$ genotypes agreed extremely well with that of the GRA6 in this study and confirmed this result (unpublished data). Type II isolates were largely predominant in human toxoplasmosis and animal infections $[8,9,12]$.

Twelve PCR products of the GRA6 gene from representative Okinawa isolates in this study were cloned and sequenced. The sequence homologies among the clones ranged from 95.3 to $100 \%$ at the nucleotide sequence and 91.3 to $100 \%$ at amino acid sequence levels, respectively. Sequence analysis of the SAG1 [2] and SAG2 [13] genes indicated a $\leq 1 \%$ sequence difference among representative strains from the three major lineages. The GRA6 gene is obviously more polymorphic than the markers commonly used for typing $T$. gondii isolates.

The GRA6 nucleotide sequences of two Okinawa isolates that were characterized as genotype II in this study were

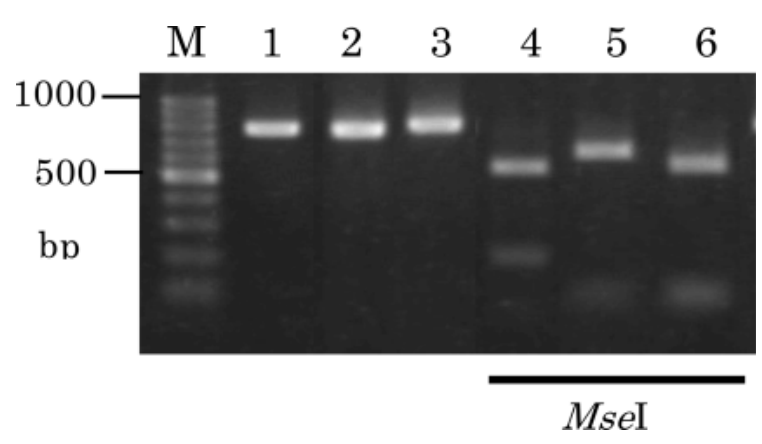

Fig. 1. Typical result of nested PCR-RFLP analysis of the GRA6 gene from $T$. gondii-infected samples. Lanes 1-3 show the PCR products of the GRA6 gene from strain types I, II, and III, respectively. Lanes 4-6 are the MseI restriction fragments of the GRA6 gene from strain types I, II, and III, respectively. $\mathrm{M}$ is the molecular-weight marker.

completely identical with the Beverley strain (typical strain II). However, 10 other clones harbored nine different sequences that had not been previously described. These nine sequences were aligned with eight sequences of the reference strains (Table 1). Nucleotide substitutions were found at 37 positions as a whole, while substitutions at 13 positions were found only for Okinawa isolates. Two deletions of $15 \mathrm{bp}$ and $3 \mathrm{bp}$ were observed in 3 Okinawa isolates, as described previously in the Beverley strain [6]. However, the $3 \mathrm{bp}$ deletion identified in the MAS strain was not found in Okinawa isolates. Thirty of 37 nucleotide substitutions resulted in no amino acid changes (Table 1). The ratio of non-synonymous to synonymous amino acid changes was not as high as that observed in a previous analysis, i.e., 21 to 22 [6]. Positive selection pressure on the GRA6 gene in $T$. gondii does not seem to be particularly strong; however, the reasons for the difference are not clear.

No nucleotide substitution was found at the potential $\mathrm{N}$ glycosylation site (codon 74; nucleotide 242-244) and putative transmembrane region at carboxyl-terminus (codons 152-171; nucleotide 476-535). However, two nucleotide substitutions, resulting in a synonymous and a non-synonymous amino acid change, were found at another putative transmembrane region at the amino-terminus (codons 2042; nucleotide 80-148).

Figure 2 shows a phylogenetic tree for the GRA6 nucleotide sequences of nine clones from Okinawa isolates, together with eight reference strains. All isolates were divided into one of three distinct groups, and two groups, including the RH strain (typical strain I) and the NED strain (typical strain III), were more closely related. The clones from the Okinawa isolates of GRA6 genotypes II and III seemed to be closely related to the Beverly and NED strains, respectively. On the other hand, the clones of genotype I were related to the typical RH strain without indicating a relationship to the other strains.

The results from this study suggest that the sequence analysis of the GRA6 gene might be useful for the molecular epidemiologic investigation of $T$. gondii infection. 
Table 1. Nucleotide polymorphism of the GRA6 gene coding region within the $T$. gondii isolates of Okinawa (shown in boldface type) and other reference strains

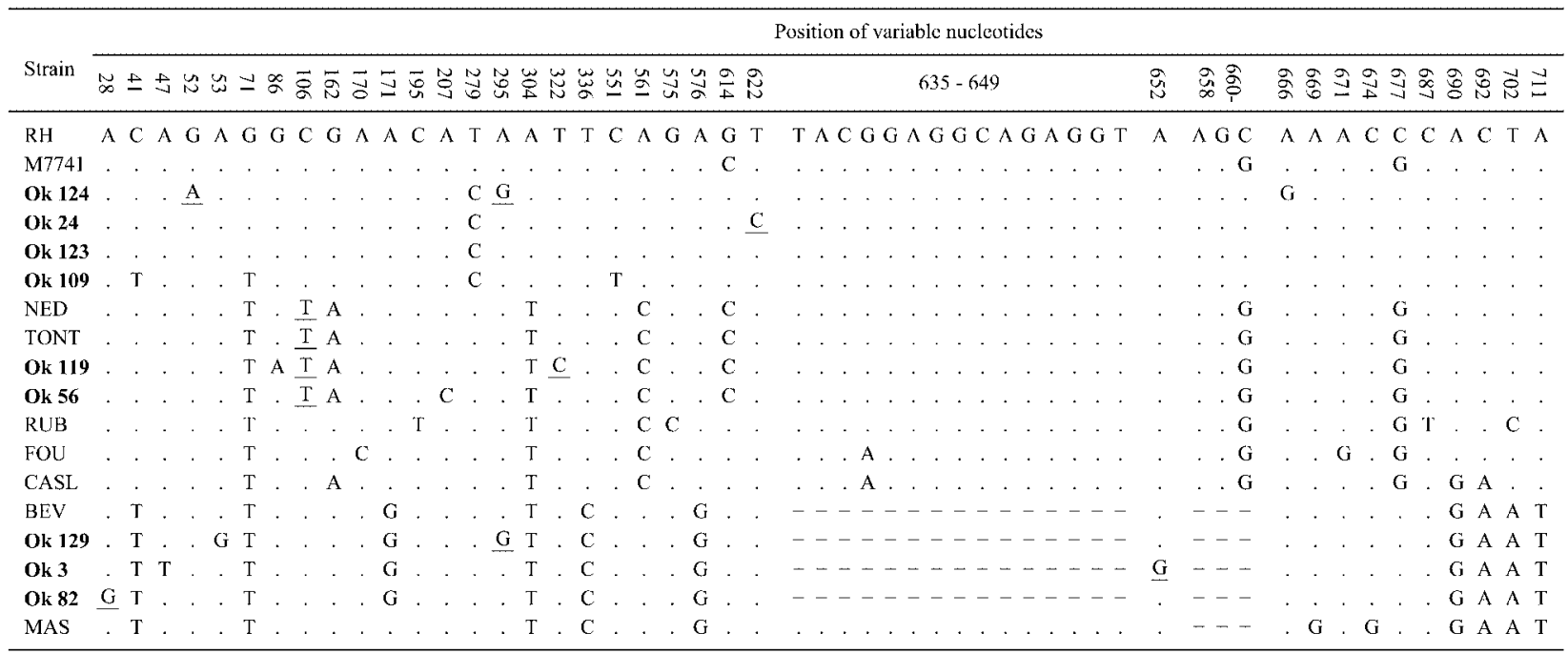

Dots (.) indicate identical nucleotides related to the sequence of RH (first row), and dashes (-) indicate deletions. The nucleotide substitutions resulting in synonymous amino acid changes are underlined. The accession numbers of the GRA6 gene nucleotide sequences are AF239283 (RH); AF239284 (BEVERLEY); AF239286 (NED); AF239287 (M7741); AF239288 (FOU); AF239289 (MAS); AF239290 (RUB); AF239291 (CASTELLS); AF239292 (TONT); AB235426 (Ok3); AB235428-AB235435 (Ok24, Ok56, Ok82, Ok109, Ok119, Ok123, Ok124, Ok129, respectively).

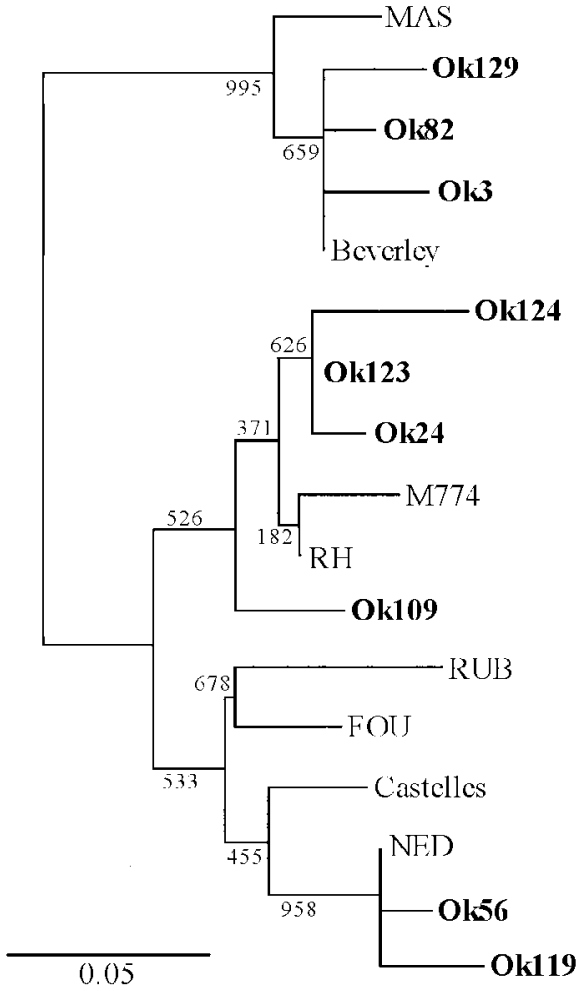

Fig. 2. The phylogenetic tree was constructed by the neighbor-joining method using the nucleotide sequences of the GRA6 genes of T. gondii of reference strains and representative Okinawa isolates (indicated in boldface type). The scale bar indicates a 5\% nucleotide difference. Bootstrap values are shown at branch nodes.

\section{REFERENCES}

1. Ajzenberg, D., Banuls, A. L., Tibayrenc, M. and Darde, M. L. 2002. Int. J. Parasitol. 32: 7-38.

2. Bulow, R. and Boothryd, J. C. 1991. J. Immunol. 147: 496500 .

3. Darde, M. L. 2004. Ann. Ist. Super Sanita 40: 57-63.

4. Desmonts, G. and Couvreur, J. 1974. New Engl. J. Med. 290: $1110-1116$.

5. Dubey, J. P. 1986. J. Am. Vet. Med. Assoc. 189: 166-170.

6. Fazaeli, A., Carter, P. E., Darde, M. L. and Pennington, T. H. 2000. Int. J. Parasitol. 30: 637-642.

7. Holland, G. N. 1999. Am. J. Ophthalmol. 128: 502-505.

8. Howe, D. K. and Sibley, L. D. 1994. Exp. Parasitol. 78: 242245.

9. Howe, D. K. and Sibley, L. D. 1995. J. Infect. Dis. 172: 15611566.

10. Howe, D. K., Honore, S., Derouin, F. and Sibley, L. D. 1997. J. Clin. Microbiol. 35: 1411-1414.

11. Lecordier, L., Moleon-Borodowsky, I., Dubremetz, J. F., Tourvieille, B., Mercier, C., Deslee, D. and Capron, A. 1995. Mol. Biochem. Parasitol. 70: 85-94.

12. Mondragon, R., Howe, D. K., Dubey, J. P. and Sibley, L. D. 1998. J. Parasitol. 84: 639-641.

13. Pamley, S. F., Gross, U., Sucharczuk, A., Windeck, T., Sgarlato, G. D. and Remington, J. S. 1994. J. Parasitol. 80: 293301.

14. Saitou, N. and Nei, M. 1987. Mol. Biol. Evol. 4: 406-425.

15. Sibley, L. D., Pfefferkorn, E. R. and Boothroyd, J. C. 1991. Parasitol. Today 7: 327-328.

16. Tenter, A. M., Heckeroth, A. R., Weiss, C. and Cowman, A. F. 2000. Int. J. Parasitol. 30: 1217-1258.

17. Thompson, J. D., Higgins, D. G. and Gibson, T. J. 1994. Nucleic. Acids Res. 22: 4673-4680. 